\section{Fabrikplanung in der Automobilindustrie}

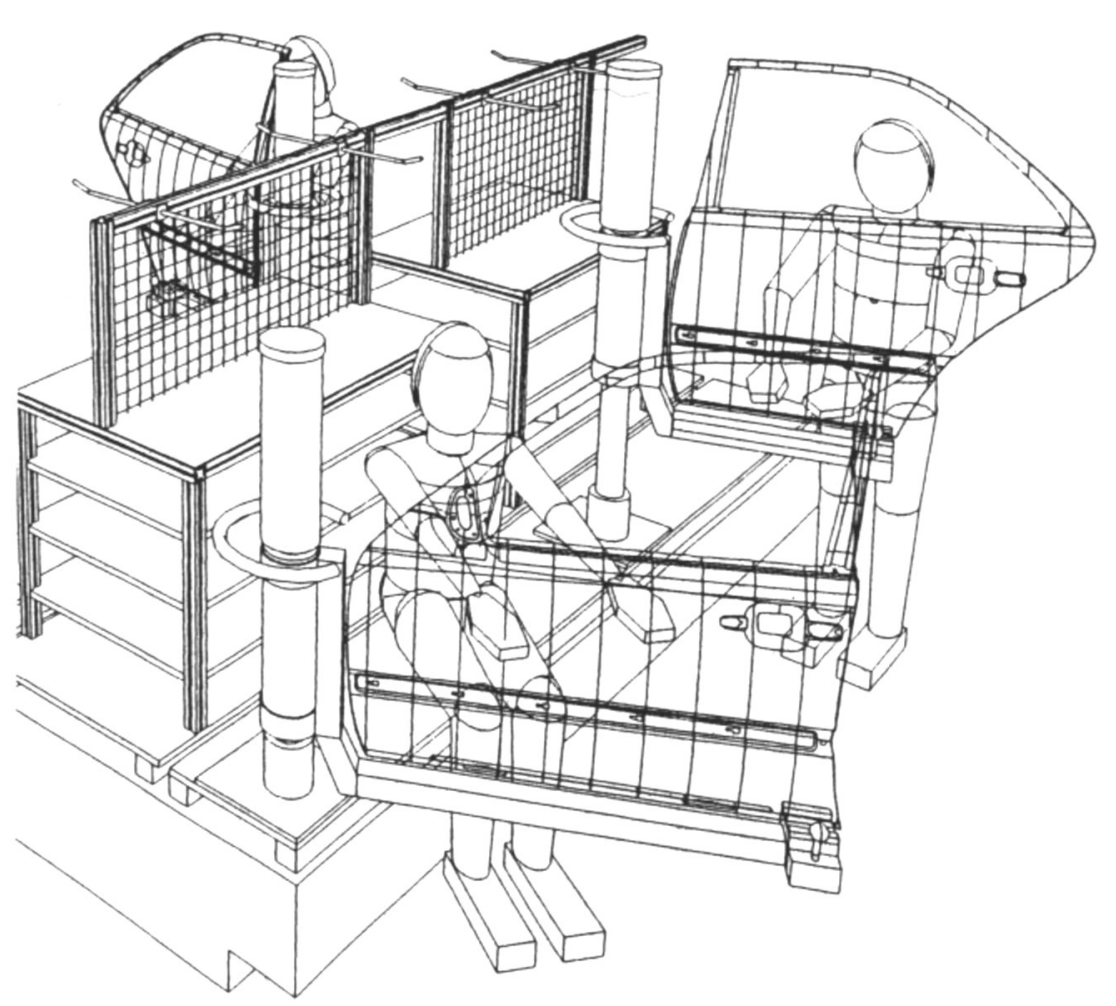

Die Bedeutung der Fabrikplanung steigt in gleichem Maße, wie sich die Innovationszyklen von Produkten, Fertigung, Logistik und Arbeitsorganisation verkürzen. Ziel des hier vorgestellten Projekts in der Automobilindustrie ist die Abdeckung des gesamten Aufgabenspektrums aller bei der Fabrikplanung beteiligten Fachbereiche von der Bau- über die Layout- bis zur Installationsplanung.

S. 298

\section{CAE-Baukastensystem für die Fabrikplanung}

Die Vielfalt von Teilsystemen zur Lösung von Planungsaufgaben erfordert eine übergeordnete Organisationsstruktur. Leitgedanken beim Aufbau des hier beschriebenen Planungssystems auf Basis einer objektorientierten Datenbank waren Transparenz, Systemoffenheit und Durchgängigkeit der Datenstruktur.

S. 304

\section{Rechnerunterstützte \\ Segmentierung im Elektro- Großmaschinenbau}

Für die Aufteilung eines Gesamtunternehmens in dezentrale, autonome Produkteinheiten (Segmente) spricht vor allem die Übertragung der marktwirtschaftlichen Ordnung auf das Unternehmen. Die Anwendung des Kunden-Lieferanten-Prinzips innerhalb eines Unternehmens führt zu einem sich weitgehend selbst regelnden System unter Berücksichtigung des Ziels einer größtmöglichen Gesamteffizienz. Die produktorientierte Aufbauorganisation ist durch einfache Strukturen, eine flache Hierarchie und eine geringe Arbeitsteilung gekennzeichnet.

\section{Editorial}

Der Mensch steht im Zentrum der Fabrikgestaltung

Notizen

aus Branchen und Unternehmen

aus der Technik.

296

zu Veranstaltungen

296

Fabrikplanung

U. Bracht, G. Friedrich, D. Schmidt: Dynamische Fabrikplanung Fertigungsprozesse simulieren

Logistikarbeitsplatz zur Fabrikplanung . . . . . . . . . . . . . . .

Zusammenhänge von komplexen Daten erkennen.

C.-A. Schumann, K. Dimitrov: Produkte, Prozesse und Fertigungsanlagen mit CAE-Baukastensystem planen .

Fertigungssysteme gestalten und verwalten . . . . . . . . . . . . . 308

CIM-orientierte Prüfstands-Automatisierung . . . . . . . . . . . . 308

Produktionsanlagen simulieren . . . . . . . . . . . . . . . 308

V. Horn, P. Trage: Segmentierung steigert die Leistung. . . . . . . . 309

Objektorientierte Datenbank im Unix-Netz . . . . . . . . . . . . . . 313

Kostengünstig automatisieren durch SPS-Dezentralisierung . . . . . 313

Neues Fertigwarenlager für Hersteller von Verpackungssystemen . 313

H. K. Tönshoff, V. Lange: Fertigungsanlagen wissensbasiert entwerfen............................ 314

Verkürzung der Durchlaufzeiten durch CAD-PPS-Kopplung . . . . . 318

Auftragseinplanung dynamisieren . . . . . . . . . . . . . . 318

U. Hallwachs: Dezentrale Verantwortungsbereiche verbinden Wirtschaftlichkeit und attraktive Arbeitsumgebung. . . . . . . . . . . 319

Neue CAD-Möglichkeiten für den Behälter- und Apparatebau . . . 323

Analyseprogramm optimiert die Bestände im Fertigwarenlager . . . 323

H. Bomm: Investitionen in Anlagen mit Ziel- und Kennzahlensystem planen.

324

\section{Fabrikanlagen und Materialfluß}

T. Elsner: Automatische Identifikation im Fertigungsfluß

Gewinde berührungslos erkennen.

H. Mutschler: Informationen in Leitwarten codieren und organisieren.

Die Mensch-Maschine-Kommunikation erfordert Bilder . . . . . . . . 335

Produktiv arbeiten mit Leitstand . . . . . . . . . . . . . . 335

H. K. Tönshoff, M. Glöckner: Chaos und Produktionsprozesse . . 336

Projektmanagement unter Windows

339

Rechneranwendung in Fertigung und Montage

K.-H. Berghäuser, F. J. Jansen: Fertigungsleitsysteme im dezentralen Einsatz . . . . . . . . . . . . . . . . . . Sicher steuern und überwachen . . . . . . . . . . . . . .

G.-A. Kemmner: Funktionsorientierte Verfahren zur Dezentralisierung von PPS-Systemen. . . . . . . . . . . . .

Report

$\mathrm{CAD} \cdot \mathrm{CAM} \cdot \mathrm{CIM}$

Fabrikanlagen und Materialfluß. 


\section{Produktionstechnisches Kolloquium \\ Berlin 1992}

21.-23. Oktober $1992 \mathrm{im} \mathrm{ICC-Berlin}$

Die wirtschaftliche Entwicklung in Europa wie auch in der gesamten Welt ist in Prozesse der Erneuerung von historischem Ausmaß eingebunden. Divergente gesellschaftliche Entwicklungen in Ost und West haben zu unterschiedlichen Wirtschaftsstrukturen geführt. Um die Grundlage für eine gesamteuropäische Zukunft zu schaffen, müssen Integrationsprozesse eingeleitet werden, die ein Zusammenwachsen unter wirtschaftlichen und technologischen, aber auch unter sozialen und ökologischen Kriterien entschlossen vorantreiben. Insbesondere die Länder Osteuropas wollen ihre Entwicklungsrückstände aufholen. Zukunftsorientierte Unternehmen müssen dem zunehmenden Wettbewerb gewachsen sein. Mit dem einheitlichen Europäischen Wirtschaftsraum verbinden sich Chancen zum Wachstum, allerdings nur dann, wenn die Unternehmen durch rechtzeitige strukturelle Anpassung ihres Leistungspotentials auf die neue Dimension vorbereitet sind.

Der Aufbruch wird gelingen, wenn die technische Weiterentwicklung der Fabrik mit einer organisatorischen Neugestaltung einhergeht. Die Arbeit mit fortgeschrittenen Technologien und veränderten Organisationsformen stellt an alle Mitarbeiter neue Anforderungen, eröffnet aber auch neue Möglichkeiten zur beruflichen Selbstverwirklichung.

Markt, Arbeit und Fabrik sind Blickrichtungen unternehmerischen Handelns, die mit gesellschaftlichen Wandlungsprozessen verbunden sind. Das Produktionstechnische Kolloquium, das in diesem Herbst zum siebten Mal stattfindet, will aufzeigen, welche Chancen und Möglichkeiten bestehen, aber auch welche Wege und Werkzeuge entwickelt werden müssen, um den notwendigen industriellen Aufbruch in Ost und West erfolgreich gestalten zu können.

In diesem Sinne gilt auch hier das Fontane-Wort: Am Mut hängt der Erfolg.

\section{Wissensbasierte Anlagenplanung}

Strukturplanung und Auslegung erfolgen heute überwiegend noch manuell. Das in diesem Beitrag beschriebene Planungssystem integriert die Funktionen Konfigurierung, Materialflußsimulation und Wirtschaftlichkeitsrechnung. Das System unterstützt den Benutzer durch einen systematischen, dialogorientierten Planungsablauf und nachvollziehbare Lösungsvorschläge. Die für die Konfigurierung notwendige objektorientierte Modellierung von Fertigungsaufgabe und -anlage schafft eine grundlegende Voraussetzung für die Integration weiterer Planungsfunktionen. Wissenserwerb und Wissensverarbeitung bei der Konfigurierung werden an einem Beispiel erläutert.

\section{Codierung von Informationen in der rechnerunterstützten} Fertigung
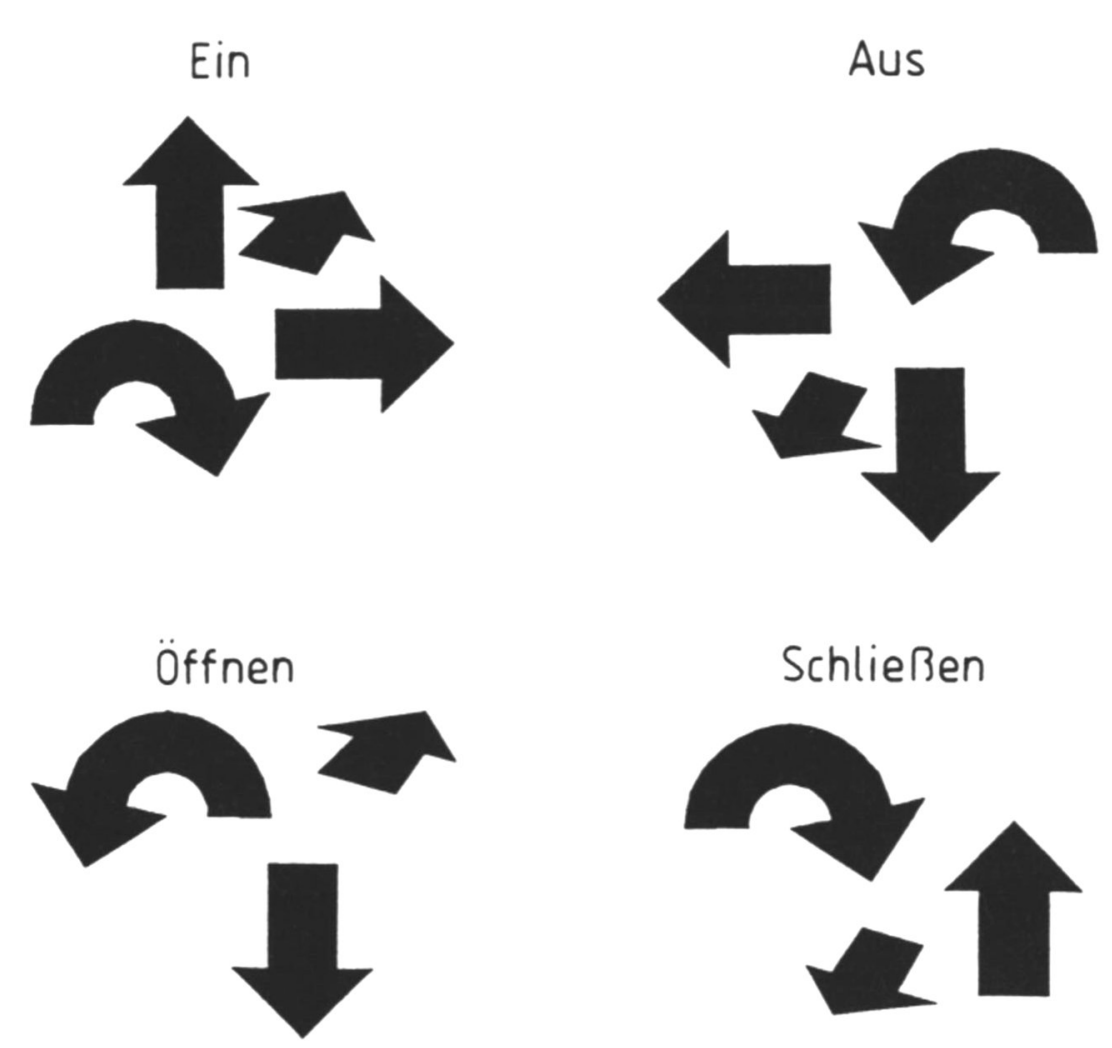

Die Wahl der Codierung für die Informationsdarstellung von Prozessen hat einen entscheidenden Einfluß auf die Qualität der Mensch-Maschine-Kommunikation. In diesem Beitrag wird die Thematik am Beispiel von Leitwarten dargestellt.

S. 332

\section{Chaosforschung in der Produktionstechnik}

Erkenntnisse darüber, unter welchen Umständen ein System zu chaotischem Verhalten neigt, spiegeln sich verstärkt in neueren Ansätzen zur Fertigungsorganisation wider. So kann eine deterministisch geplante Werkstatt prinzipiell eher zu unvorhersehbaren Störungen führen, während ein statistisches Modell einen stabileren Prozeß ermöglicht.

S. 336 